\title{
Bayes Shrinkage Estimation of the Parameter of Rayleigh Distribution for Progressive Type-II Censored Data
}

\author{
Sanku Dey \\ St. Anthony's College
}

\author{
Tanujit Dey \\ Cleveland Clinic \\ (corresponding author)
}

\author{
Sudhansu S. Maiti \\ Visva-Bharati University
}

\begin{abstract}
This paper derives Bayes shrinkage estimator of Rayleigh parameter and its associated risk function based on conjugate prior under the assumption of general entropy loss function for progressive Type-II censored data. Risk function of maximum likelihood estimate, Bayes estimate and Bayes shrinkage estimate have also been derived and compared. An empirical Bayes estimate procedure has been suggested to include a guess value in case of the Bayes shrinkage estimation. Risk function of empirical Bayes estimate and empirical Bayes shrinkage estimate have also been derived and compared. In conclusion, an illustrative example is presented to assess how the Rayleigh distribution fits a real data set.
\end{abstract}

Keywords: Bayes estimate, conjugate prior, empirical Bayes estimate, Rayleigh distribution, risk function.

\section{Introduction}

Rayleigh distribution first introduced in the literature by Lord Rayleigh (1980) has been widely used in reliability theory and survival analysis because of it's flexibility and simplicity. An important characteristic of the Rayleigh distribution is that its failure rate is a linear function of time. The reliability function of Rayleigh distribution decreases at a much higher rate than the reliability function of exponential distribution. The probability density function of one parameter Rayleigh distribution has the form:

$$
f(x \mid \theta)=\frac{x}{\theta} \exp \left(-\frac{x^{2}}{2 \theta}\right) ; \quad x>0, \theta>0 .
$$

and the cumulative density function (cdf) has the form:

$$
F(x \mid \theta)=1-\exp \left(-\frac{x^{2}}{2 \theta}\right) ; \quad x>0, \theta>0 .
$$

In recent years, several authors have carried out extensive studies in relation to the estimation, prediction and other inferences with respect to Rayleigh distribution. Sinha and Howlader 
(1983) obtained credible and highest posterior density (HPD) intervals of the parameter and reliability of Rayleigh distribution. Ariyawansa and Templeton (1984) have also discussed some of its applications. Howlader (1985) obtained HPD prediction intervals for the Rayleigh distribution. Howlader and Hossain (1995) obtained Bayes estimators for the scale parameter and the reliability function in the case of Type-II censored sampling. Abd Elfattah, Hassan, and Ziedan (2006) studied the efficiency of the maximum likelihood estimates of the parameter under three cases, namely, Type-I, Type-II and progressive Type-II censored sampling schemes. Wu, Chen, and Chen (2006) obtained Bayes estimators and highest posterior density credible intervals for parameter and reliability function of the Rayleigh distribution, as well as the Bayes predictive estimator and prediction interval for future observations based on progressively Type-II censored samples. Hendi, Abu-Youssef, and Alraddadi (2007) obtained Bayes estimators of the scale parameter, reliability function and failure rate by using non-informative prior and Hartigan prior based on upper record values. Dey and Das (2007) obtained Bayesian predictive intervals of the parameter of Rayleigh distribution. Dey (2009) also obtained Bayes estimators for the parameter and reliability function of the Rayleigh distribution under different loss function. Dey (2009) also studied the Bayes estimators for the parameter and reliability function of Rayleigh distribution based on complete as well as Type-II censored samples, also compared relative risk functions.

Our key role is to obtain Bayes shrinkage estimator for the parameter of Rayleigh distribution, which is different than the approaches referenced above. The shrinkage estimator are valuable as in many practical situations, the experimenter has some prior information about the parameter value in the form of a point guess value and that value can be used to make inference of the parameter. In this condition, our parameter of interest is $\theta$ and thereby the guess value $\theta_{0}$ can be used to make inference for $\theta$. In this article, we use an empirical Bayes estimation procedure for $\theta_{0}$ based on sample observation.

Several authors have considered the use of the point guess value for inferences with regard to the parameter. For instance; Prakash and Singh (2006) studied shrinkage estimators for the inverse dispersion for inverse Gaussian distribution under LINEX loss function. Singh, Prakash, and Singh (2007) studied shrinkage estimators for the shape parameter of Pareto distribution using the LINEX loss function. Singh, Singh, Singh, and Upadhyay (2008) studied the Bayes estimators of the failure rate and reliability function for a one-parameter exponential distribution by utilizing a point guess estimate of the parameter. Prakash (2009) obtained some shrinkage estimators and the Bayes estimators for the shape parameter of a Pareto distribution under the general entropy loss function. Al-Hemyari and Al-Dabag (2012) studied a class of shrinkage estimators for the shape parameter of the Weibull lifetime model. Salman and Shareef (2014) studied preliminary test single stage Bayesian shrinkage estimator for the scale parameter of an exponential distribution under the improper prior distribution using the quadratic loss function.

Perhaps the most popular technique that utilizes the point guess value is the shrinkage technique, originally suggested by Thompson (1968). The shrinkage estimator performs better than the usual estimator when a guess value is approximately the true value of the parameter given the sample size is small. Thompson (1968) considered the problem of shrinking an unbiased estimator $\hat{\zeta}$ of the parameter $\zeta$ toward a natural origin $\zeta_{0}$ and suggested a shrinkage type estimator $k \hat{\zeta}+(1-k) \zeta_{0}$, where $k(0<k<1)$ is a constant.

The shrinkage technique has been utilized in numerous studies namely; mean survival time in epidemiological studies (Harris and Shakarki 1979), projecting the money supply (Tso 1990), estimating mortality rates (Marshall 1991) and improving estimation in sample surveys (Wooff 1985). In life-testing and reliability experiments, units that are subject to test are sometimes lost or removed from the experiment before failure. Such units are usually called the censored units.The most common censoring schemes are Type-I censoring and Type-II censoring but one of the drawbacks of the conventional Type-I and Type-II censoring schemes is that they are inflexible in removing units at that point of execution but rather at the end of the experiment. One censoring scheme known as progressive Type-II censoring scheme 
overcomes this shortcoming and this has led to its popularity in recent years.

\subsection{Review of progressive Type-II sampling}

Progressive censoring is useful in both industrial life testing applications and clinical settings; it allows the removal of surviving experimental units before the termination of the test. Balakrishnan and Aggarwala (2000) provided a comprehensive reference on the subject of progressive censoring and its applications. For further reading, the readers are referred to Kundu (2008), Kundu and Pradhan (2009), Ng, Kundu, and Chan (2009) and the references cited therein. A schematic representation of progressively Type-II right censored sample is depicted in Figure 1.1 (Cramer and Iliopoulos 2010).

Under this censoring scheme, $n$ units are placed on a test at time zero and $m$ failures are to be observed. When the first failure is observed, $r_{1}$ of surviving units are randomly selected and removed from the experiment. At the time of second failure, $r_{2}$ of the remaining $n-r_{1}-1$ units are randomly selected and removed from the experiment. Finally, at the $m$ th failure all the remaining surviving units $r_{m}=n-m-r_{1}-r_{2}-\ldots-r_{m-1}$ are removed from the experiment. In this censoring scheme, $r_{1}, r_{2}, \ldots, r_{m}$ are all prefixed.

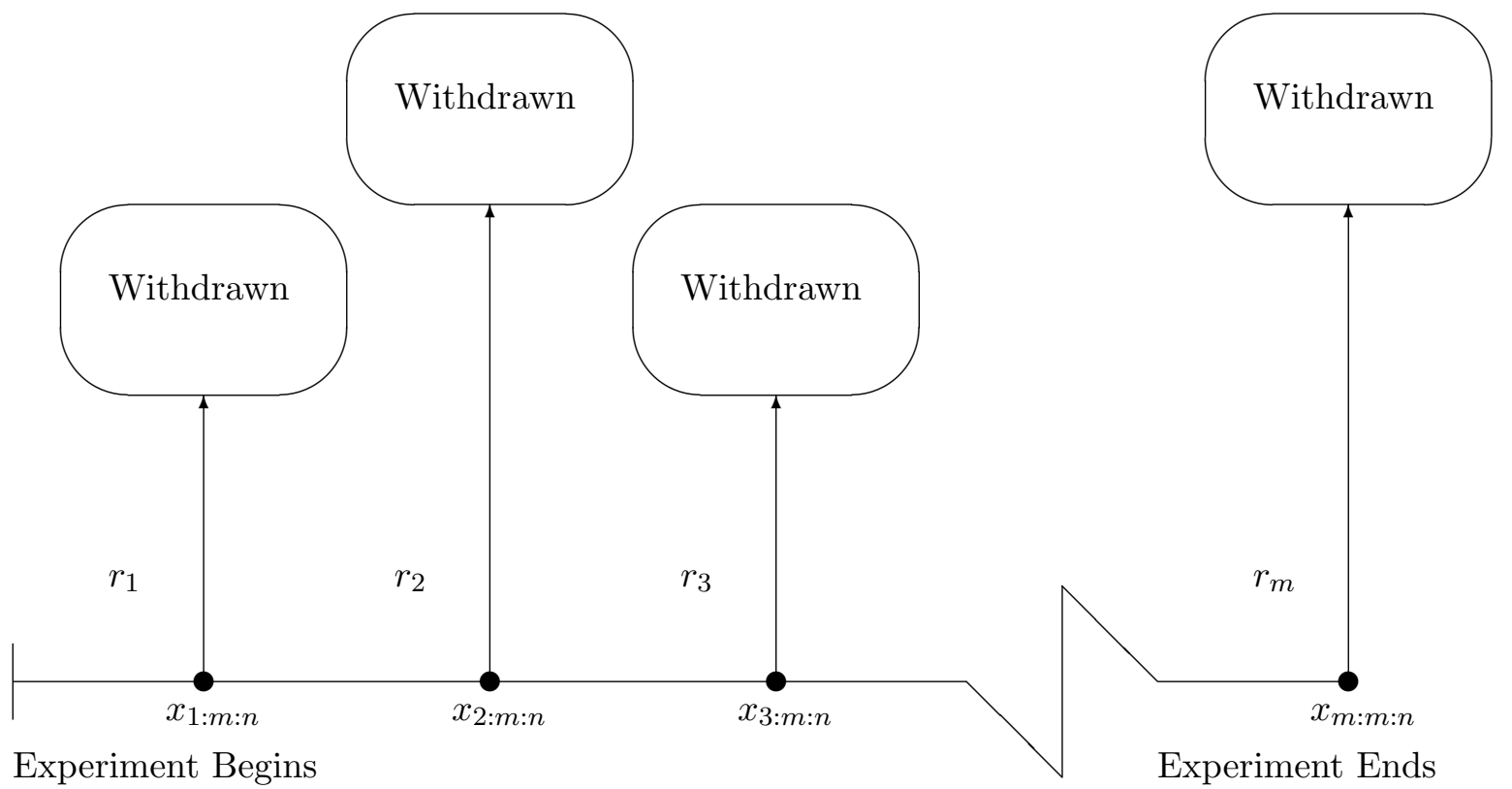

Figure 1: Schematic representation of a progressively Type-II right censored sample where $x_{1: m: n}, x_{2: m: n}, \ldots, x_{m: m: n}$ denote the observed failure times and $r_{1}, r_{2}, \ldots, r_{m}$ denote the corresponding numbers of items removed (withdrawn) from the test.

The key goal of our article is to obtain Bayes estimator and Bayes shrinkage estimator for the parameter of Rayleigh distribution with conjugate prior distribution based on progressively Type-II censored samples. Since we have no idea about the true value of the parameter $\theta$ and thus the guess value $\theta_{0}$, so we propose to obtain the empirical Bayes estimate of $\theta_{0}$ on the basis of sample observation to approximate the guess value $\theta_{0}$. Unlike the publications cited above where the focus is on obtaining shrinkage estimator based on complete or TypeII censored samples using exponential, Pareto or Weibull distribution, we have considered Rayleigh distribution with progressive Type-II censoring schemes. Moreover, an empirical Bayes estimate has been taken as guess value for obtaining Bayes shrinkage estimates. Also, risk function of empirical Bayes estimate and empirical Bayes shrinkage estimate have been 
obtained for comparison.

The rest of the paper is organized as follows. Section 2 discusses prior and loss function used in our Bayesian estimation. In Section 3, we obtained the Bayes' estimators of $\theta$ and risk function of maximum likelihood estimator, Bayes estimator and Bayes shrinkage estimator. A simulation study is performed in Section 4, and a real life data is used in Section 5 for the evaluation of classical Bayes estimate and Bayes shrinkage estimate and their estimated risks. We conclude the paper in Section 6.

\section{Prior and loss function}

In the Bayesian approach, $\theta$ is considered a random variable with some specified distribution. In this paper, we consider conjugate prior distribution of the form

$$
g(\theta \mid \alpha, \beta) \propto \theta^{-(\alpha+1)} \exp \left(-\frac{\beta}{\theta}\right), \theta>0,
$$

where $\alpha>0$ and $\beta>0$. The advantage of using natural conjugate prior is that the resulting posterior distribution will also belong to the same family. Furthermore, Jeffreys' prior can be obtained as a special case of (3) by substituting $\alpha=\beta=0$ and Hartigan's prior can be obtained by substituting $\alpha=2, \beta=0$.

In many practical situations it is more realistic to express the loss in terms of ratio $\hat{\theta} / \theta$. In this case, Calabria and Pulcini (1996) proposed a loss function, the general entropy loss function of the form:

$$
L(\hat{\theta}, \theta) \propto\left[\left(\frac{\hat{\theta}}{\theta}\right)^{p}-p \ln \left(\frac{\hat{\theta}}{\theta}\right)-1\right] ; \quad p \neq 0,
$$

whose minimum occurs at $\hat{\theta}=\theta$. This loss is a generalization of the entropy loss used by several authors [see, for example, Dey, Ghosh, and Srinivasan (1987) and Dey and Liu (1992)], where the shape parameter $p$ is taken to be equal to 1 . If we assume, $\ln \left(\frac{\hat{\theta}}{\theta}\right)=$ $\hat{\theta}-\theta$ i.e., $\left(\frac{\hat{\theta}}{\theta}\right)=e^{(\hat{\theta}-\theta)}$, we get the linear exponential (LINEX) loss function of the form, $\left[e^{p(\hat{\theta}-\theta)}-p(\hat{\theta}-\theta)-1\right]$ which is proposed by Zellner (1986). Following Calabria and Pulcini (1996), the Bayes estimators for the parameter $\theta$ given data $\underline{x}$ under general entropy loss function (GELF) may be defined as

$$
\hat{\theta}_{G B}=\left[E\left(\theta^{-p} \mid \underline{x}\right)\right]^{-\frac{1}{p}} .
$$

\section{Estimation}

Let $X=\left(X_{1: m: n}, X_{2: m: n}, \ldots, X_{m: m: n}\right)$ be a progressively Type-II right censored sample from a life test of effective sample size $m$ from a sample of size $n$, where the ordered lifetimes have a Rayleigh distribution with pdf and cdf as given by (1) and (2) with a pre-determined number of removal of units from the test, say $R_{1}=r_{1}, R_{2}=r_{2}, \ldots, R_{m}=r_{m}$. For convenience, we write $X_{i: m: n}$ as $X_{(i)}$, the $i$ th ordered lifetime. Here the likelihood function of $\theta$ is given by

$$
\begin{aligned}
l(\theta) & =A \cdot \prod_{i=1}^{m} f\left(x_{(i)} \mid \theta\right)\left[1-F\left(x_{(i)} \mid \theta\right)\right]^{r_{i}}, \text { where } A=n\left(n-r_{1}-1\right) \ldots\left(n-\sum_{j=1}^{m-1} r_{j}-m+1\right) \\
& =A \cdot \theta^{-m} e^{-\frac{1}{2 \theta} \sum_{i=1}^{m}\left(1+r_{i}\right) x_{(i)}^{2}} \prod_{i=1}^{m} x_{(i)} \\
& =A \cdot \theta^{-m} e^{-\frac{T}{2 \theta}} \prod_{i=1}^{m} x_{(i)},
\end{aligned}
$$


where $T=\sum_{i=1}^{m}\left(1+r_{i}\right) x_{(i)}^{2}$, and $x_{(i)}$ is the observation of $X_{(i)} ; i=1,2, \ldots m$. Here the maximum likelihood estimate of $\theta$ is $\hat{\theta}_{M L E}=\frac{T}{2 m}$. It is to be noted that $\hat{\theta}_{M L E}$ is an unbiased estimator of $\theta$.

Lemma 3.1 Under progressive Type-II censoring, $\frac{T}{\theta}$ follows a chi-square distribution with degrees of freedom $2 m$.

Proof: Let $Z_{i}=\frac{X_{(i)}^{2}}{2 \theta} ; i=1, \ldots, m$, then $Z_{1}<Z_{2}<\ldots<Z_{m}$ is a progressive Type-II censored sample from the standard exponential distribution. Considering the following transformations

$W_{1}=n Z_{1}$

$W_{2}=\left(n-r_{1}-1\right)\left(Z_{2}-Z_{1}\right)$

$W_{3}=\left(n-r_{1}-r_{2}-2\right)\left(Z_{3}-Z_{2}\right)$

$W_{m}=\left(n-r_{1}-r_{2}-\ldots-r_{m-1}-m+1\right)\left(Z_{m}-Z_{m-1}\right)$

Hence $W_{1}, W_{2}, \ldots, W_{m}$ are all independent and identically distributed as standard exponential. Then we have

$2 \sum_{i=1}^{m} W_{i} \sim \chi_{2 m}^{2}$, that is

$2 \sum_{i=1}^{m} W_{i}=2 \sum_{i=1}^{m}\left(1+r_{i}\right) Z_{i}=2 \sum_{i=1}^{m}\left(1+r_{i}\right) \frac{X_{(i)}^{2}}{2 \theta}=\frac{T}{\theta} \sim \chi_{2 m}^{2}$.

Combining the prior density (3) with the likelihood function (5) and by using Bayes theorem the joint posterior distribution is derived as follows:

$$
\pi(\theta \mid T)=\frac{\left(\beta+\frac{T}{2}\right)^{m+\alpha} \theta^{-(m+\alpha+1)} e^{-\frac{1}{\theta}\left(\beta+\frac{T}{2}\right)}}{\Gamma(m+\alpha)} .
$$

The posterior distribution of $\theta$ is inverse gamma with parameters $(m+\alpha)$ and $\left(\beta+\frac{T}{2}\right)$. Under the general entropy loss function, the Bayes estimator of $\theta$ is the posterior expectation

$$
\begin{aligned}
\hat{\theta}_{G B} & =\left[E\left(\theta^{-p} \mid T\right)\right]^{-\frac{1}{p}} \\
& =\left[\int_{0}^{\infty} \theta^{-p} \pi(\theta \mid T) d \theta\right]^{-\frac{1}{p}} .
\end{aligned}
$$

On simplification we get,

$$
\hat{\theta}_{G B}=\left[\frac{\Gamma(m+\alpha)}{\Gamma(m+\alpha+p)}\right]^{\frac{1}{p}}\left(\beta+\frac{T}{2}\right) .
$$

We choose the parameters of the prior distribution $g(\theta)$ such that $E\left(\hat{\theta}_{G B}\right)=\theta_{0}$, where $\theta_{0}$ is the point guess value of $\theta$. This gives

$$
E\left(\hat{\theta}_{G B}\right)=\theta_{0} \quad \Rightarrow \quad \beta=\left(\frac{1}{c}-m\right) \theta_{0}
$$

where $c=\left[\frac{\Gamma(m+\alpha)}{\Gamma(m+\alpha+p)}\right]^{\frac{1}{p}}$.

Substituting this value of $\beta$ in $\hat{\theta}_{G B}$, we obtain the Bayes shrinkage estimator for $\theta$ under GELF as

$$
\hat{\theta}_{G B}^{S}=c \cdot m \cdot T_{1}+(1-c \cdot m) \theta_{0}=k_{1} \cdot T_{1}+\left(1-k_{1}\right) \theta_{0},
$$

where, $k_{1}=c \cdot m=\left[\frac{\Gamma(m+\alpha)}{\Gamma(m+\alpha+p)}\right]^{\frac{1}{p}} m$ and $T_{1}=\frac{T}{2 m}\left(=\hat{\theta}_{M L E}\right)$. 
The pdf of $W=\frac{\hat{\theta}_{G B}}{\theta}=a(\theta)+b \cdot \frac{T}{\theta}$ is given by

$$
f_{W}(w)=\left\{\begin{array}{l}
\frac{1}{b \cdot 2^{m} \Gamma(m)} e^{-\frac{1}{2}\left(\frac{w-a(\theta)}{b}\right)}\left(\frac{w-a(\theta)}{b}\right)^{m-1}, \quad w>a(\theta), \\
0, \quad \text { elsewhere, }
\end{array}\right.
$$

where $a(\theta)=\frac{c \cdot \beta}{\theta}$ and $b=\frac{c}{2}$.

The pdf of $V=\frac{\hat{\theta}_{G B}^{S}}{\theta}=e(\theta)+d \cdot \frac{T}{\theta}$ is given by

$$
f_{V}(v)=\left\{\begin{array}{lc}
\frac{1}{d \cdot 2^{m} \Gamma(m)} e^{-\frac{1}{2}\left(\frac{v-e(\theta)}{d}\right)}\left(\frac{v-e(\theta)}{d}\right)^{m-1}, \quad v>e(\theta) \\
0, \quad \text { elsewhere, }
\end{array}\right.
$$

where, $e(\theta)=\frac{\left(1-k_{1}\right) \theta_{0}}{\theta}$ and $d=\frac{k_{1}}{2 m}$.

The risk function of $\hat{\theta}_{G B}$ under GELF is given by

$$
\begin{aligned}
R_{G B}(\theta)= & E\left[L\left(\hat{\theta}_{G B}, \theta\right)\right] \\
= & E\left[\left(\frac{\hat{\theta}_{G B}}{\theta}\right)^{p}-p \ln \left(\frac{\hat{\theta}_{G B}}{\theta}\right)-1\right] \\
= & E\left[\left(a(\theta)+b \cdot \frac{T}{\theta}\right)^{p}-p \ln \left(a(\theta)+b \cdot \frac{T}{\theta}\right)-1\right] \\
= & \sum_{j=0}^{p} \frac{p(p-1) \ldots(p-j+1)}{j !} a(\theta)^{j}(2 b)^{p-j} \frac{\Gamma(m+j)}{\Gamma(m)} \\
& -p \int_{0}^{\infty} \ln (a(\theta)+b \cdot w) \cdot \frac{1}{2^{m} \Gamma(m)} e^{-\frac{w}{2}} w^{m-1} d w-1 \text { for } p>0 .
\end{aligned}
$$

Similarly, we will have the risk function of $\hat{\theta}_{G B}^{S}$ under GELF as $R_{G B S}(\theta)$ and is given by

$$
\begin{aligned}
R_{G B S}(\theta)= & E\left[\left(\frac{\hat{\theta}_{G B}^{S}}{\theta}\right)^{p}-p \ln \left(\frac{\hat{\theta}_{G B}^{S}}{\theta}\right)-1\right] \\
= & E\left[\left(e(\theta)+d \cdot \frac{T}{\theta}\right)^{p}-p \ln \left(e(\theta)+d \cdot \frac{T}{\theta}\right)-1\right] \\
= & \sum_{j=0}^{p} \frac{p(p-1) \ldots(p-j+1)}{j !}\left(\frac{\left(1-k_{1}\right) \theta_{0}}{\theta}\right)^{j}\left(\frac{k_{1}}{m}\right)^{p-j} \frac{\Gamma(m+j)}{\Gamma(m)} \\
& -p \int_{0}^{\infty} \ln \left(\left(\frac{\left(1-k_{1}\right) \theta_{0}}{\theta}\right)+\left(\frac{k_{1}}{2 m}\right) \cdot w\right) \cdot \frac{1}{2^{m} \Gamma(m)} e^{-\frac{w}{2}} w^{m-1} d w-1 \text { for } p>0 .
\end{aligned}
$$

The risk function of $\hat{\theta}_{M L E}$ under GELF is given by

$$
\begin{aligned}
R_{M L E}(\theta) & =E\left[\left(\frac{\hat{\theta}_{M L E}}{\theta}\right)^{p}-p \ln \left(\frac{\hat{\theta}_{M L E}}{\theta}\right)-1\right] \\
& =\frac{\Gamma(m+p)}{\Gamma(m)}-p[\psi(m)-\ln (2 m)]-1 \text { for } p>0,
\end{aligned}
$$

where $\psi($.$) is the digamma function.$

To find the Bayes shrinkage estimator, we need a guess value of $\theta_{0}$ that at times could be unknown. We will use the empirical Bayes estimate of $\theta$ in place of $\theta_{0}$. The Bayes' estimator given in (6) depends on $\alpha$ and $\beta$, and that in (7) depends on $\alpha$, which are the parameters of the prior distribution of $\theta$. The parameters $\alpha$ and $\beta$, could be estimated by means of empirical Bayes' procedure (see Lindley (1969) and Awad and Gharraf (1986)). Given the 
random sample $\underline{x}$, the likelihood function of $\theta$ has an inverse gamma density with shape parameter $(m-1)$ and scale parameter $\frac{T}{2}$. Following this, we can use the estimates of the prior parameters $\alpha$ and $\beta$ from the sample by $(m-1)$ and $\frac{T}{2}$ respectively. Hence we can take $\hat{\theta}_{0}$ as

$$
\begin{aligned}
\hat{\theta}_{0}=\hat{\theta}_{G B . e m p} & =\left[\frac{\Gamma(2 m-1)}{\Gamma(2 m+p-1)}\right]^{\frac{1}{p}} T \\
& =c_{e m p} \cdot T
\end{aligned}
$$

with $c_{e m p}=\left[\frac{\Gamma(2 m-1)}{\Gamma(2 m+p-1)}\right]^{\frac{1}{p}}$.

To find out $\hat{\theta}_{G B S . e m p}$, we substitute $\hat{\theta}_{0}$ in place of $\theta_{0}$ and hence

$$
\begin{aligned}
\hat{\theta}_{G B S . e m p} & =c_{e m p} \cdot m \cdot T_{1}+\left(1-c_{e m p} \cdot m\right) \hat{\theta}_{0} \\
& =k_{1 . e m p} \cdot T_{1}+\left(1-k_{1 . e m p}\right) \hat{\theta}_{0} \\
& =k_{1 . e m p} \cdot \hat{\theta}_{M L E}+\left(1-k_{1 . e m p}\right) \hat{\theta}_{0} \\
& =\left(\frac{3}{2} c_{e m p}-m \cdot c_{e m p}^{2}\right) T=k_{e m p} T .
\end{aligned}
$$

The risk functions of empirical Bayes estimator and empirical Bayes shrinkage estimator are

$$
R_{G B . e m p}(\theta)=\frac{\left(2 c_{e m p}\right)^{p} \cdot \Gamma(m+p)}{\Gamma(m)}-p\left[\psi(m)+\ln \left\{2 c_{e m p}\right\}\right]-1
$$

and

$$
R_{G B S . e m p}(\theta)=\frac{\left(2 k_{e m p}\right)^{p} \cdot \Gamma(m+p)}{\Gamma(m)}-p\left[\psi(m)+\ln \left\{2 k_{e m p}\right\}\right]-1
$$

respectively.

\section{Simulation study}

We present some experimental results to observe the performance of Bayes estimation of $\theta$ using the prior (3) for different sample sizes, different effective sample sizes, different priors, and for different sampling schemes. We have considered different sample sizes $n=20,25,30,35$, different effective sample sizes; $m=10,15$, and 20, different hyper-parameter values for $\alpha$ and $\beta$, and six ([1] - [6]) different sampling schemes as described in Table 1. We have simulated progressive Type-II samples using the algorithm proposed by Balakrishnan and Sandhu (1995) from the Rayleigh model with $\theta=2$. The estimators are compared based on the average value of estimates and their corresponding risk performances. For Bayesian computation, we have considered two different set of hyperparameters: $\alpha=\beta=0$, which is a non-informative prior (labeled as "Prior 1") and $\alpha=\beta=2$, which is an informative prior (labeled as "Prior 2"). The two different values of $p$ are considered for the loss function: $p=1,2$. The simulation results are summarized in Table 2 and 3. All results are based on 10,000 repetitions.

From Tables 2 and 3 we notice that risk does not depend on sample size $n$ but it depends on effective sample size $m$, and as effective sample size increases, the risk decreases and average Bayes estimates come closer to the true value of $\theta$. When a guess value $\theta_{0}$ is chosen in the neighborhood of $\theta$, then obviously the risk is minimum and Bayes shrinkage estimate is better. But in practice, $\theta$ is unknown and choosing a guess value is difficult one. Even though the risk for empirical Bayes estimates are a bit more, but from a pragmatic point of view, empirical Bayes shrinkage estimator is worthwhile. 
Table 1: Different progressive Type-II censoring schemes used in the simulation study

\begin{tabular}{|c|c|}
\hline Number & Scheme \\
\hline$[1]$ & $\left(\begin{array}{llllllllll}0 & 0 & 0 & 0 & 0 & 0 & 0 & 0 & 0 & 10\end{array}\right)$ \\
\hline$[2]$ & $\left(\begin{array}{llllllllll}0 & 0 & 0 & 0 & 0 & 0 & 0 & 0 & 0 & 15\end{array}\right)$ \\
\hline$[3]$ & 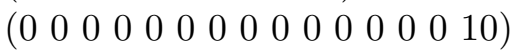 \\
\hline$[4]$ & 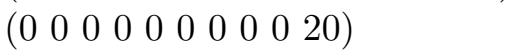 \\
\hline$[5]$ & $\left(\begin{array}{lllllllllllllll}0 & 0 & 0 & 0 & 0 & 0 & 0 & 0 & 0 & 0 & 0 & 0 & 0 & 0 & 15\end{array}\right)$ \\
\hline$[6]$ & 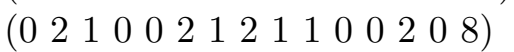 \\
\hline
\end{tabular}

* In the Table, Scheme $\left(\begin{array}{llll}0 & 0 & 0 & r\end{array}\right)$ indicates that at 1st, 2nd and 3rd failure, no active unit is withdrawn or removed but at 4 th failure, $r$ active units are withdrawn or removed from the test.

Table 2: The average MLE and the Bayes estimators $(\hat{\theta})$ from different estimation procedures for the parameter $\theta$ under the general entropy loss function with $p=1$. The true risk (in bold) and the estimated risk are presented within parentheses.

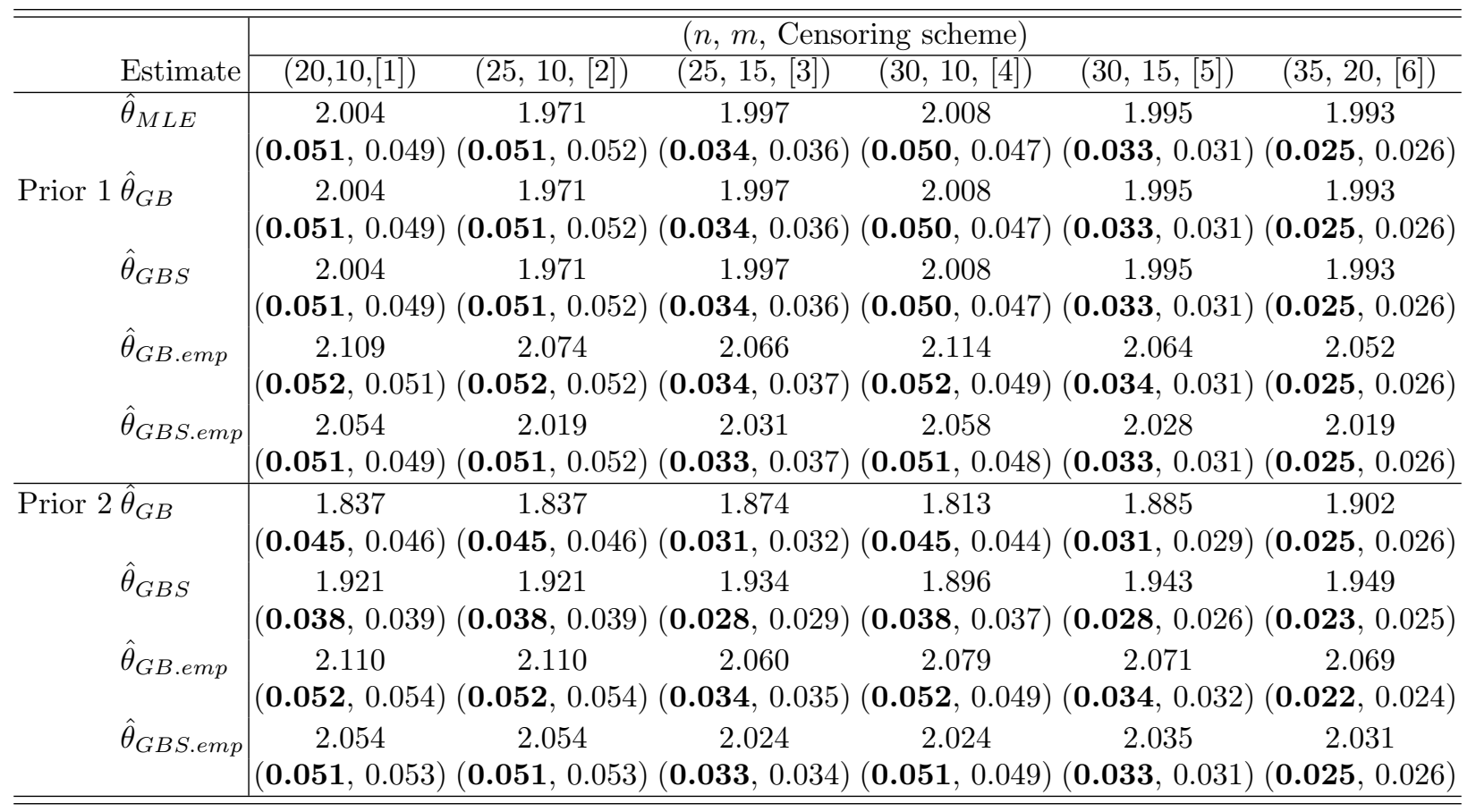

\section{Data analysis}

We are considering the data that appeared in tests on endurance of deep grove ball bearings [Lawless (1982), p.228]. The data are the number of hundreds of million revolutions before failure for each of the 23 ball bearings in the life test:

$0.1788,0.2892,0.33,0.4152,0.4212,0.456,0.4848,0.5184,0.5196,0.5412,0.5556,0.678$, $0.6864,0.6864,0.6888,0.8412,0.9312,0.9864,1.0512,1.0584,1.2792,1.2804,1.734$.

To study the goodness of fit of the Rayleigh model, we compute the $\chi^{2}$ statistic (with 3 degrees of freedom) and it is 1.0312 with the corresponding p-value 0.7937 . Therefore, the high $p$-value clearly suggests that the one parameter Rayleigh model can be used to analyze this data set. Besides this, we also plotted the scaled TTT (TTT stands for "total time on test") transformed (Aarset 1987) of the ball bearing data. Usually this plot is used to identify whether a random sample is from a lifetime distribution with constant against bathtub-type hazard rate; for further details see Aarset (1987). Figure 2 indicates that the empirical hazard function is unimodal and therefore it is reasonable to use Rayleigh distribution to analyze the 
Table 3: The average MLE and the Bayes estimators $(\hat{\theta})$ from different estimation procedures for the parameter $\theta$ under the general entropy loss function with $p=2$. The true risk (in bold) and the estimated risk are presented within parentheses.

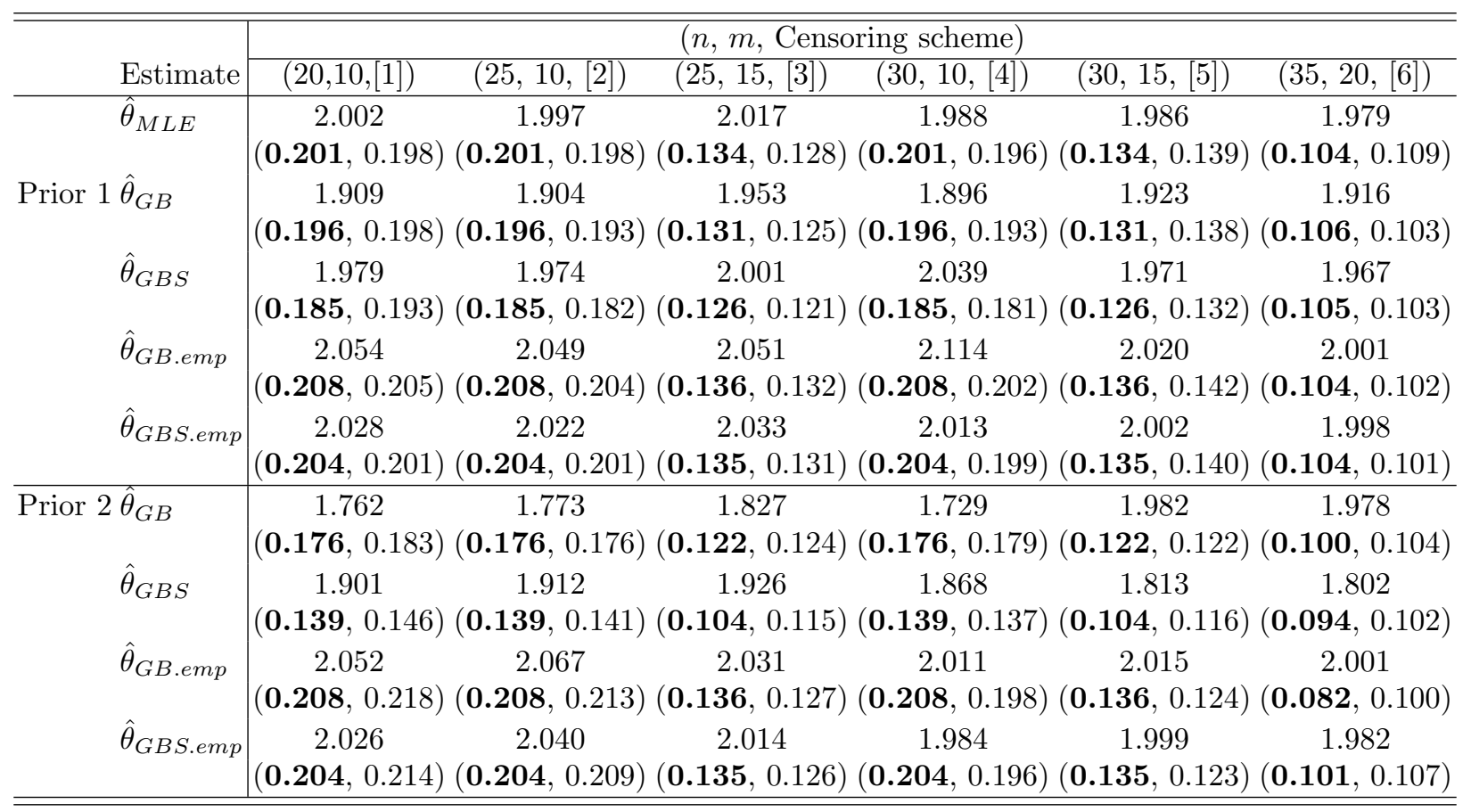

data.

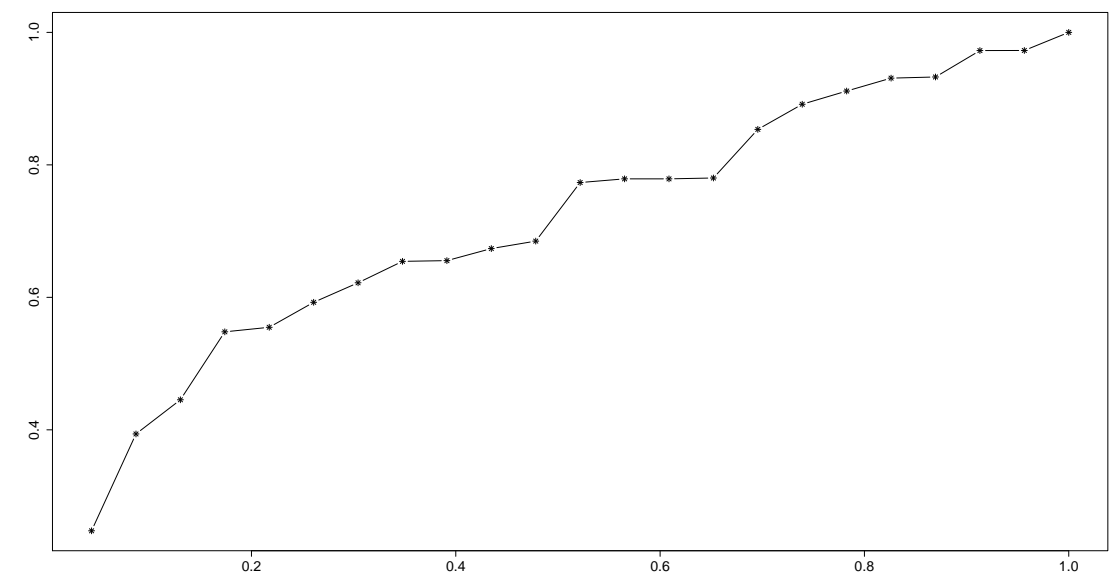

Figure 2: Scaled TTT transformed plot of the ball bearing data

For the purposes of illustrating the methods discussed in this article, a progressively Type-II censored sample was generated from this data set with effective sample size $m=13$. The observations (in hundreds of millions) and removed numbers are reported in Table 4 [see Wu et al. (2006)].

It is observed from Table 5 that the Empirical Bayes Shrinkage Estimate of $\theta$ is better in terms of minimum risk sense. As $p$ increases, estimates decrease and their risks increase. 
Table 4: Progressively Type II censored sample for Example.

\begin{tabular}{lccccrrrr}
\hline$i$ & 1 & 2 & 3 & 4 & 5 & 6 & 7 \\
$x_{(i)}$ & 0.1788 & 0.2892 & 0.33 & 0.4212 & 0.456 & 0.4848 & 0.5184 \\
$r_{i}$ & 0 & 0 & 3 & 0 & 0 & 2 & 0 \\
\hline$i$ & 8 & 9 & 10 & 11 & 12 & 13 \\
$x_{(i)}$ & 0.5196 & 0.6780 & 0.6864 & 0.8412 & 0.9312 & 1.2792 \\
$r_{i}$ & 0 & 2 & 0 & 2 & 1 & 0 \\
\hline
\end{tabular}

Table 5: Empirical Bayes and Empirical Bayes Shrinkage Estimate and their risks for the ball bearing data.

\begin{tabular}{lllll}
\hline$p$ & $\begin{array}{l}\text { Empirical Bayes } \\
\text { Estimate }\end{array}$ & $\begin{array}{l}\text { Empirical Bayes } \\
\text { risk }\end{array}$ & $\begin{array}{l}\text { Empirical Bayes } \\
\text { Shrinkage Estimate }\end{array}$ & $\begin{array}{l}\text { Empirical Bayes } \\
\text { Shrinkage risk }\end{array}$ \\
\hline 1 & 0.3809 & 0.0397 & 0.3733 & 0.0391 \\
\hline 2 & 0.3735 & 0.1586 & 0.3698 & 0.1565 \\
\hline \hline
\end{tabular}

\section{Conclusion}

In this article, we have derived Bayes shrinkage estimate of the parameter of the Rayleigh distribution under conjugate prior assuming general entropy loss function. The Bayes estimate, Bayes shrinkage estimate assuming a point guess value are derived and their risks have been studied. Bayes shrinkage estimate is reasonably good from a risk perspective. In practice, as the true value of the parameter is unknown, getting a point guess value is difficult. An empirical Bayes procedure has been followed to get an estimated guess value of the parameter and utilizing so, empirical Bayes and empirical Bayes shrinkage estimates and their risks have been calculated. The performance of the empirical Bayes shrinkage estimate is fairly reasonable and competitive. We recommend empirical Bayes shrinkage estimate for practical purposes intended for enhanced outcomes. Real life data analysis also echos similar trend as observed in the simulation study.

\section{Acknowledgments}

The authors would like to thank the editor and the reviewer who helped to substantially improve the paper.

\section{References}

Aarset M (1987). "How to Identify a Bathtub Hazard Function." IEEE Transactions on Reliability, 36, 106-108.

Abd Elfattah AM, Hassan AS, Ziedan DM (2006). "Efficiency of Maximum Likelihood Estimators under Different Censored Sampling Schemes for Rayleigh Distribution." Interstat. URL http://interstat. statjournals . net/YEAR/2006/articles/0603001.pdf.

Al-Hemyari ZA, Al-Dabag HA (2012). "A Class of Shrinkage Testimators for the Shape Parameter of the Weibull Lifetime Model." Pakistan Journal of Statistics and Operational Research, 8(2), 167-184. 
Ariyawansa KA, Templeton JGC (1984). "Structural Inference on the Parameter of the Rayleigh Distribution from Doubly Censored Samples." Statist. Hefte, 25, 181-199.

Awad AM, Gharraf MK (1986). "Estimation of $P[Y<X]$ in the Burr Case: A Comparative Study." Communications in Statistics- Simulation and Computation, 15(15), 189-203.

Balakrishnan N, Aggarwala R (2000). Progressive Censoring: Theory, Methods and Applications. Birkhäuser, Boston.

Balakrishnan N, Sandhu RA (1995). "A Simple Simulation Algorithm for Generating Progressive Type-II Censored Samples." The American Statistician, 49(2), 119-230.

Calabria R, Pulcini G (1996). "Point Estimation under Asymmetric Loss Functions for LeftTruncated Exponential Samples." Communications in Statistics-Theory 85 Methods, 25(3), 585-600.

Cramer E, Iliopoulos G (2010). "Adaptive Progressive Type-II Censoring." Test, 19(2), 342358.

Dey DK, Ghosh M, Srinivasan C (1987). "Simultaneous Estimation of Parameters under Entropy Loss." Journal of Statistical Planning and Inference, 15, 347-363.

Dey DK, Liu PL (1992). "On Comparison of Estimators in a Generalized Life Model." Microelectronics Reliability, 32, 207-221.

Dey S (2009). "Comparison of Bayes Estimators of ohe Parameter and Reliability Function for Rayleigh Distribution under Different Loss Functions." Malaysian Journal of Mathematical Sciences, 3, 247-264.

Dey S, Das MK (2007). "Note on Prediction Interval for a Rayleigh Distribution: Bayesian Approach." American Journal of Mathematical and Management Science, 1-2, 43-48.

Harris E, Shakarki G (1979). "Use of the Population Distribution to Improve Estimation of Individual Mean in Epidemiological Studies." Journal of Chronical Disease, 32, 233-243.

Hendi ML, Abu-Youssef SE, Alraddadi AA (2007). "A Bayesian Analysis of Record Statistics from the Rayleigh Model." International Mathematical Forum, 2(13), 619-631.

Howlader HA (1985). "HPD Prediction Intervals for Rayleigh Distribution." IEEE Transactions on Reliability, 34, 121-123.

Howlader HA, Hossain A (1995). "On Bayesian Estimation and Prediction from Rayleigh Distribution Based on Type-II Censored Data." Comm. Stat. Theory Methods, 24(9), $2249-2259$.

Kundu D (2008). "Bayesian Inference and Reliability Sampling Plan for Weibull Distribution." Technometrics, 50, 144-154.

Kundu D, Pradhan B (2009). "Bayesian Inference and Life Testing Plans for Generalized Exponential Distribution." Science in China, Series A: Mathematics, 52(6), 1373-1388.

Lawless JF (1982). Statistical Models and Methods for Lifetime Data. John Wiley and Sons, New York.

Lindley DV (1969). Introduction to Probability and Statistics from a Bayesian View Point. Vol.1. Cambridge University Press.

Marshall RJ (1991). "Mapping Disease and Mortality Rates using Empirical Bayes Estimators." Journal of Applies Statistics, 40, 283-294. 
Ng HKT, Kundu D, Chan PS (2009). "Statistical Analysis of Exponential Lifetimes under an Adaptive Type-II Progressive Censoring Scheme." Naval Research Logistics, 56, 687-698.

Prakash G (2009). "Some Estimators for the Pareto Distribution." Journal of Scientific Research, 1(2), 236-247.

Prakash G, Singh DC (2006). "Shrinkage Estimators for the Inverse Dispersion of the Inverse Gaussian Distribution under the LINEX Loss Function." Austrian Journal of Statistics, 35(4), 463-470.

Rayleigh JWS (1980). "On the Resultant of a Large Number of Vibrations of the Some Pitch and of Arbitrary Phase." Philosophical Magazine, 5-th Series, 10, 73-78.

Salman AN, Shareef RA (2014). "Bayesian Shrinkage Estimator for the Scale Parameter of Exponential Distribution under Improper Prior Distribution." International Journal of Statistics and Applications, 4(3), 135-143.

Singh DC, Prakash G, Singh P (2007). "Shrinkage Estimators for the Shape Parameter of Pareto Distribution using the LINEX Loss Function." Communication in Statistics - Theory and Methods, 36(4), 741-753.

Singh GP, Singh SK, Singh U, Upadhyay SK (2008). "Bayes Estimators of Exponential Parameters from a Censored Sample using a Guess Estimate." Data Science Journal, 7, $106-114$.

Sinha SK, Howlader HA (1983). "Credible and HPD Intervals of the Parameter and Reliability of Rayleigh Distribution." IEEE Transactions on Reliability, 32, 283-294.

Thompson JR (1968). "Some Shrinkage Techniques for Estimating the Mean." Journal of American Statistical Association, 63, 113-122.

Tso G (1990). "Forecasting money supply in Hong Kong with a multiple shrinkage estimator." In Proceeding of the ASA Section on Business and Commerce. ASA.

Wooff A (1985). "Bounds on Reciprocal Moments with Applications and Developments in Stein Estimation and Post Stratification." Journal of Royal statistical Society, B, 47, 362371.

Wu SJ, Chen DH, Chen ST (2006). "Bayesian Inference for Rayleigh Distribution under Progressive Censored Sample." Applied Stochastic Models in Business and Industry, 22, 269-279.

Zellner A (1986). "Bayesian Estimation and Prediction using Asymmetric Loss Function." Journal of American statistical Association, 81, 446-451.

\section{Affiliation:}

Sanku Dey

Department of Statistics

St. Anthony's College

Shillong, Meghalaya, India

E-mail: sanku_dey2k2003@yahoo.co.in 
Tanujit Dey

Department of Quantitative Health Sciences

Cleveland Clinic

Cleveland, OH USA

E-mail: deyt@ccf.org

Sudhansu S. Maiti

Department of Statistics

Visva-Bharati University

Santiniketan, West Bengal, India

E-mail: dssm10rediffmail.com

\section{Austrian Journal of Statistics}

published by the Austrian Society of Statistics

Volume 44

December 2015 http://www.ajs.or.at/

http://www.osg.or.at/

Submitted: 2014-09-15

Accepted: 2015-01-27 